\title{
Improved prognosis of low baseline neutrophil-to-lymphocyte ratio is significantly exclusive in breast cancer patients with high absolute counts of lymphocytes
}

\author{
YUKIE FUJIMOTO, HIROMI OZAWA, TOMOKO HIGUCHI, YOSHIMASA MIYAGAWA, \\ AYAKO BUN, MICHIKO IMAMURA and YASUO MIYOSHI \\ Department of Surgery, Division of Breast and Endocrine Surgery, Hyogo College of Medicine, \\ Nishinomiya, Hyogo 663-8501, Japan
}

Received May 29, 2018; Accepted November 12, 2018

DOI: $10.3892 / \mathrm{mco} .2018 .1783$

\begin{abstract}
Although the neutrophil-to-lymphocyte ratio (NLR) is a valuable prognostic factor for early breast cancer, the patient subgroups that may benefit the most from NLR analysis remain unknown. The present study analyzed the prognostic significance of NLR according to absolute lymphocyte counts (ALCs). A total of 889 patients with operated early breast cancers were retrospectively recruited. Existing NLR and ALC baseline data from the time-period prior to operation or preoperative chemotherapy were collected. The cut-off value for NLR was set at 2.72 according to the receiver operating characteristic curve. Recurrence-free survival (RFS) of NLR-low patients at baseline $(n=582)$ was significantly better than that of NLR-high patients $(n=307$, $\mathrm{P}=0.036$ ). Improved patient prognoses were observed in the NLR-low, ALC-high (>1,688/ $\mu 1$; 5-year RFS, 0.88 vs. 0.57; $\mathrm{P}<0.0001)$ subgroup $(\mathrm{n}=355)$, but not in the NLR-low, ALC-low $(\leq 1,688 / \mu 1$; 5 -year RFS, 0.87 vs. $0.87 ; \mathrm{P}=0.46)$ subgroup $(\mathrm{n}=534)$. Using multivariate analysis, NLR was observed to be a significant and independent factor for RFS (hazard ratio: 3.52; 95\% confidence interval: 1.61-7.32; $\mathrm{P}=0.0023$ ) in the ALC-high breast cancer subgroup. Prognostic significance for baseline NLR was found exclusively in the ALC - high subgroup. Since NLR is a simple marker, the results obtained here might be useful for identifying patients who have high recurrence risk, and those that are candidates for additional treatments.
\end{abstract}

Correspondence to: Professor Yasuo Miyoshi, Department of Surgery, Division of Breast and Endocrine Surgery, Hyogo College of Medicine, 1-1 Mukogawa-cho, Nishinomiya, Hyogo 663-8501, Japan

E-mail: ymiyoshi@hyo-med.ac.jp

Key words: breast cancer, neutrophil-to-lymphocyte ratio, absolute lymphocyte count, prognosis

\section{Introduction}

Identification of high-risk patients with operated breast cancers is a crucial issue, as their prognoses may be expected to improve with additional adjuvant therapy. It is well established that peripheral inflammation markers, including neutrophil-to-lymphocyte ratio (NLR) and platelet-to-lymphocyte ratio are significantly associated with prognoses in early breast cancers (1-3). According to a meta-analysis of 15 studies comprising a total of 8,563 patients, high NLR was associated with both decreased disease-free survival (DFS) and worse overall survival (OS), with hazard ratio (HR) and 95\% confidence interval (CI) of 1.74; 1.47-2.07, $\mathrm{P}<0.001$ for DFS and 2.56; 1.96-3.35, $\mathrm{P}<0.001$ for OS (2). Similarly, poorer DFS (HR 1.72, 95\% CI: 1.30-2.27) and OS (HR 1.87, 95\% CI: 1.41-2.48) were reported in a meta-analysis of 18 studies (3).

Although details of the mechanisms underlying these peripheral markers for patient prognosis remain unknown, a tight connection between breast cancers and inflammation, which includes not only a protective role against cancer cells but also promotes tumor progression, is speculated. Most anti-tumor effects are mediated by cytotoxic $\mathrm{T}$ lymphocytes. In line with their protective effect, breast cancers with higher counts of tumor-infiltrating lymphocytes (TILs) are correlated with favorable prognoses (4). High numbers of peripheral lymphocytes, therefore, seem to be associated with good prognoses. In contrast, it is speculated that cancer-related inflammation, which is associated with neutrophil accumulation, is essential for tumor progression (5). Regional neutrophils produce many factors linked with chemokines, cytokines, and vascular endothelial growth factors (6). As these factors contribute to cancer cell proliferation and invasion, or to angiogenesis, their effects promote cancer progression and metastasis. Considering the role mentioned above, high peripheral counts of neutrophils are likely to result in unfavorable prognoses for breast cancer patients. Based on these hypotheses, therefore, the establishment of NLR as a prognostic measure for breast cancer is proposed.

In clinical settings, breast cancers are divided into subtypes according to estrogen receptor (ER) and human epidermal 
A Recurrence-free survival (NLR)

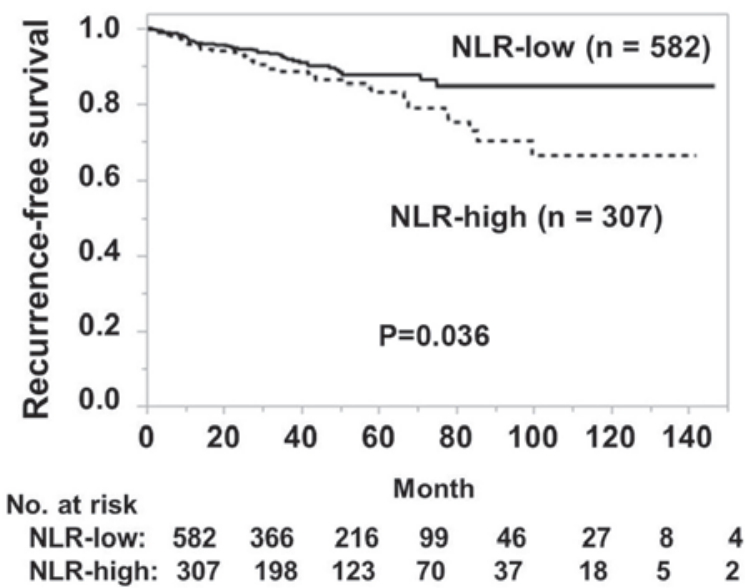

B Overall survival (NLR)

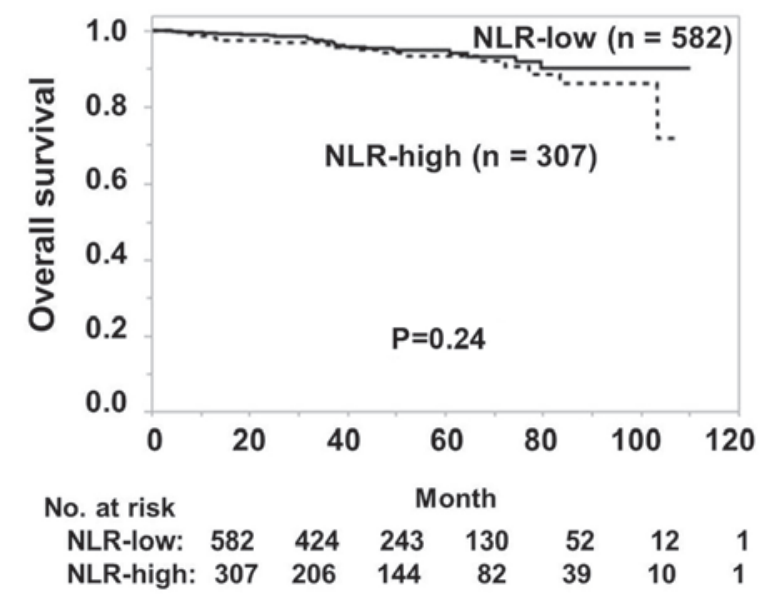

Figure 1. RFS and OS of patients according to their NLR. (A) The RFS of NLR. NLR-low patients had significantly better survival than the NLR-high patients $(\mathrm{P}=0.036)$. (B) OS was not associated with NLR levels $(\mathrm{P}=0.24)$. RFS, recurrence-free survival; OS, overall survival; NLR, neutrophil-to-lymphocyte ratio.

growth factor receptor type 2 (HER2) (7). The association between highNLR and poor prognoses is reported to be strongest in the ER-negative/HER2-negative subtype (2). Since TILs are more frequently observed in ER-negative/HER2-negative $(8,9)$, antitumor immune responses are estimated to occur frequently in this subtype. Systemic inflammation, judged by peripheral markers, may be influenced by the occurrence of regional reactions to breast cancer. The observation that the association between NLR and a patient's prognosis is strongest in the ER-negative/HER2-negative subtype (10) thus seems to indicate a connection between NLR and immunity, at least in part.

Interestingly, a high absolute lymphocyte count (ALC) was significantly associated with improved prognosis of solid tumor patients (11) and non-small cell lung cancer patients (12) treated with programmed cell death 1 (PD-1) checkpoint inhibitors. These data suggest that a high peripheral ALC serves as an indicator for cancer immunity and less immune effects are induced in patients with a low ALC. Although NLR has been clearly identified as a good prognostic indicator for breast cancer, its prognostic capability may vary depending on the ALC. Whether the use of NLR as a prognostic factor depends on the ALC or not remains to be studied. The purpose of this study was to analyze the prognostic significance of the NLR in relation to the ALC in breast cancer patients.

\section{Materials and methods}

Patient eligibility. Patients with invasive breast cancer who were operated upon at the Hyogo College of Medicine between May 2005 and December 2016 were consecutively recruited for this retrospective study. Those for whom we obtained peripheral neutrophil and lymphocyte count baseline data before operation or preoperative chemotherapy (889 of 964 patients) were considered eligible for the current study. Adjuvant chemotherapies and endocrine therapies were administered in 263 and 665 patients, respectively, with treatments based on the St Gallen guidelines at that time (7,13-17).

The median follow-up time was 30.7 months (range:1-146 months). During the follow-up, 84 patients

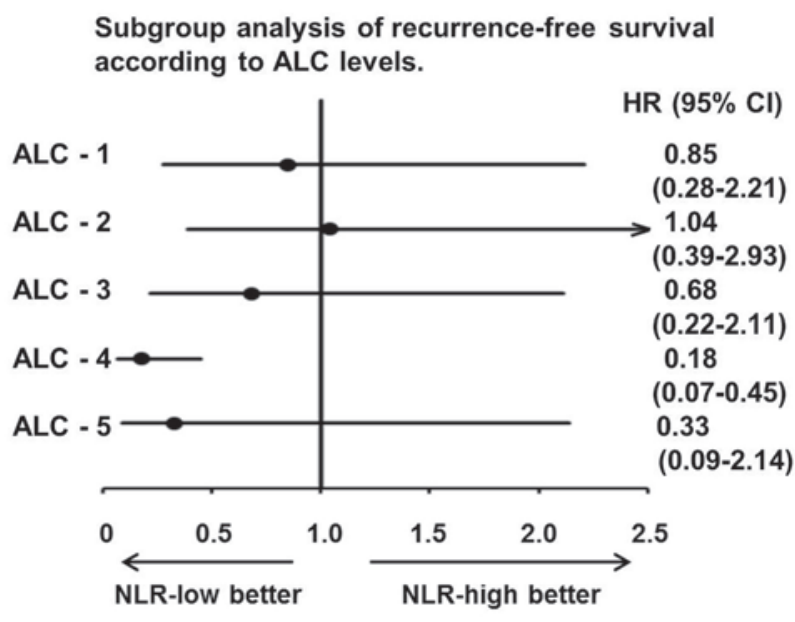

Figure 2. RFS of patients with high and low NLR in subgroups according to the ALC. HRs and 95\% CIs in each subgroup according to ALC levels divided into five equal subgroups are shown. The RFS of patients with NLR-low was better in the subgroups with the second highest $(>1,688$ and $\leq 1,977 / \mu 1)$ and highest $(>1,977 / \mu 1)$ ALCs. The central line indicates the HR 1.00. HR, hazard ratio; ALC, absolute lymphocyte count; CI, confidence interval; RFS, recurrence-free survival; OS, overall survival; NLR, neutrophil-to-lymphocyte ratio.

had recurrence, with metastases documented in bone $(\mathrm{n}=30)$, locoregional and lymph nodes $(\mathrm{n}=27)$, lungs $(\mathrm{n}=16)$, liver $(n=11)$, pleura $(n=2)$, brain $(n=8)$ and elsewhere $(n=13)$. The recurrence-free survival (RFS) was defined as the time from the operation to either the first recurrence, or to patient death by any cause. The OS was calculated from the date of operation to that of death by any cause. This study was approved by the Ethics Committee of the Hyogo College of Medicine (no. 1886) in accordance with the Declaration of Helsinki. As this study collected only retrospective clinical data and offered no risk to the participants, written informed consent was not required.

Measurements of NLR and ALC. Baseline NLR and ALC levels were determined using blood samples collected prior to 
A Recurrence-free survival (ALC)

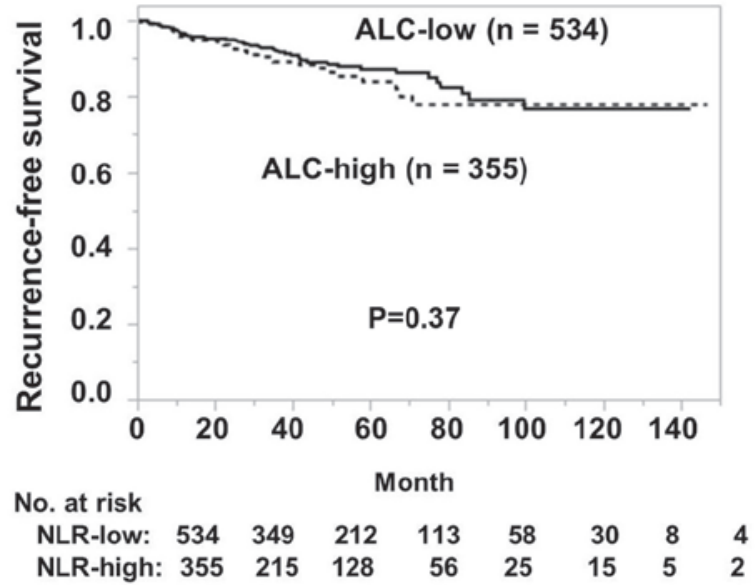

B Overall survival (ALC)

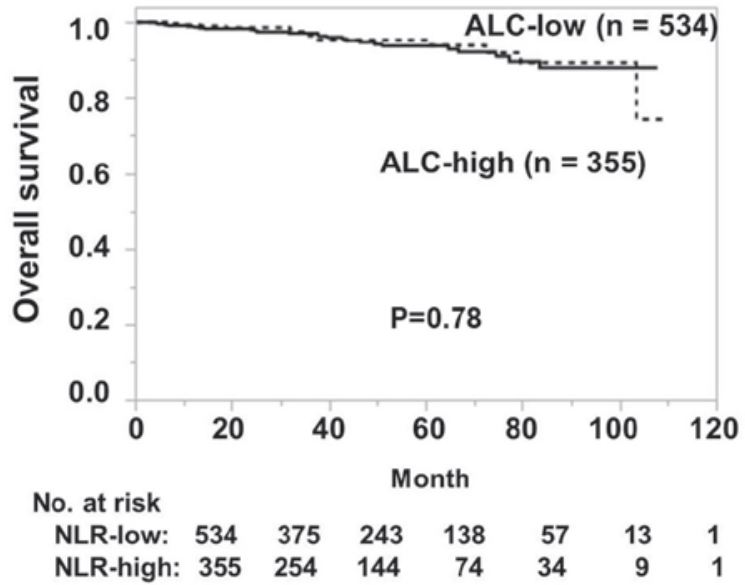

Figure 3. RFS and OS of patients according to the levels of ALC. (A) There was no significant difference in the RFS between the ALC-high and -low patients $(\mathrm{P}=0.37)$. (B) OS was not different between the ALC-high and -low patients $(\mathrm{P}=0.78)$. ALC, absolute lymphocyte count; RFS, recurrence-free survival; OS, overall survival; NLR, neutrophil-to-lymphocyte ratio.

A Recurrence-free survival (ALC-low)

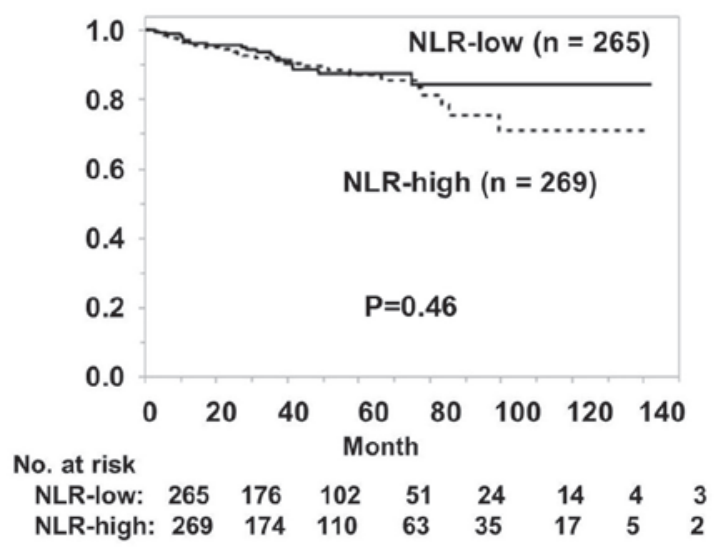

C Overall survival (ALC-low)

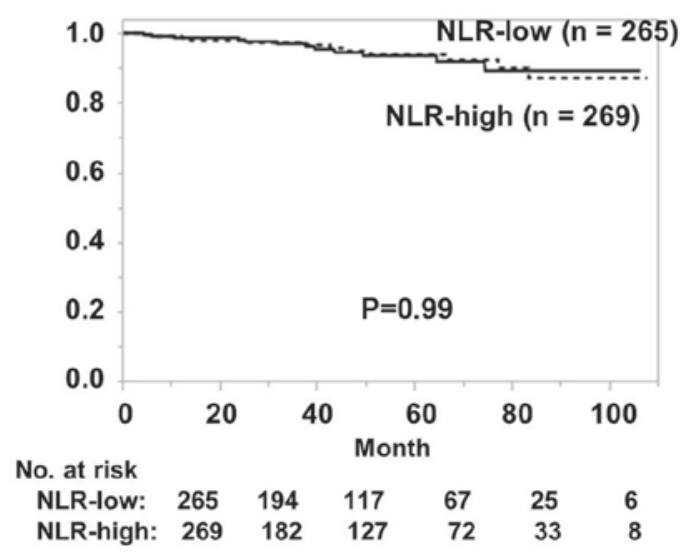

B Recurrence-free survival (ALC-high)

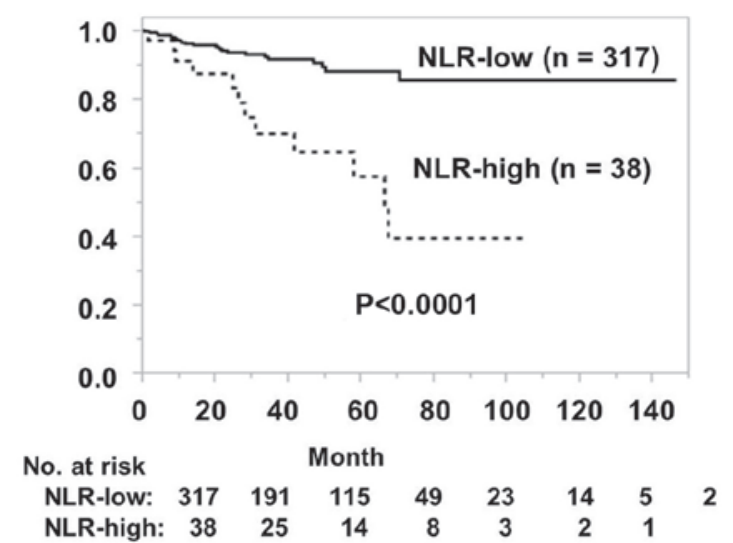

D Overall survival (ALC-high)

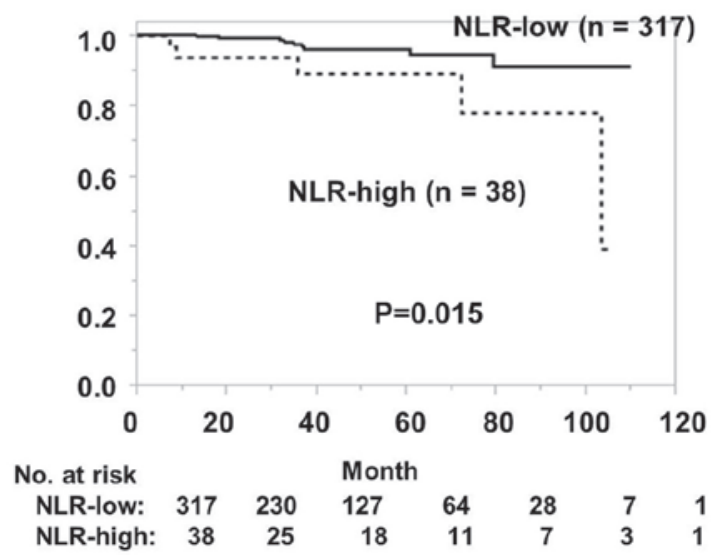

Figure 4. RFS and OS of patients according to the levels of NLR in the ALC-low and -high subgroups. There was no significant difference between NLR-low and -high patients in regard to (A) RFS and (C) OS in the ALC-low $(\leq 1,688 / \mu 1)$ subgroup $(\mathrm{P}=0.46$ and $\mathrm{P}=0.99)$. The (B) RFS and (D) OS of patients with NLR-low were significantly better than that of NLR-high in the ALC-high $(>1,688 / \mu 1)$ subgroup $(\mathrm{P}<0.0001$ and $\mathrm{P}=0.015)$. ALC, absolute lymphocyte count; RFS, recurrence-free survival; OS, overall survival; NLR, neutrophil-to-lymphocyte ratio. 
Table I. Clinicopathological characteristics of breast cancers according to NLR or ALC levels.

\begin{tabular}{|c|c|c|c|c|c|c|}
\hline Characteristics & $\begin{array}{c}\text { NLR-high }^{\mathrm{a}} \\
(\mathrm{n}=307) ; \mathrm{n},(\%)\end{array}$ & $\begin{array}{c}\text { NLR-low } \\
(\mathrm{n}=582) ; \mathrm{n},(\%)\end{array}$ & P-value & $\begin{array}{c}\text { ALC-high }^{\mathrm{b}} \\
(\mathrm{n}=355) ; \mathrm{n},(\%)\end{array}$ & $\begin{array}{c}\text { ALC-low } \\
(\mathrm{n}=534) ; n,(\%)\end{array}$ & P-value \\
\hline Menopausal status & & & 0.022 & & & 0.0012 \\
\hline Pre- & $121(39.3)$ & $187(60.7)$ & & $100(32.5)$ & $208(67.5)$ & \\
\hline Post- & $181(31.6)$ & $392(68.4)$ & & $251(43.8)$ & $322(56.2)$ & \\
\hline Unknown & $5(62.5)$ & $3(37.5)$ & & $4(50.0)$ & $4(50.0)$ & \\
\hline Tumor size, $\mathrm{cm}$ & & & 0.021 & & & 0.63 \\
\hline$\leq 2$ & $166(31.6)$ & $360(68.4)$ & & $207(39.4)$ & $319(60.7)$ & \\
\hline$>2$ & $140(39.1)$ & $218(60.9)$ & & $147(41.1)$ & $211(58.9)$ & \\
\hline Unknown & $1(20.0)$ & $4(80.0)$ & & $1(20.0)$ & $4(80.0)$ & \\
\hline Lymph node metastasis & & & 0.33 & & & 0.41 \\
\hline Negative & $201(33.7)$ & $395(66.3)$ & & $244(40.9)$ & $352(59.1)$ & \\
\hline Positive & $101(37.1)$ & $171(62.9)$ & & $103(37.9)$ & $169(62.1)$ & \\
\hline Not examined & $5(23.8)$ & $16(76.2)$ & & $8(38.1)$ & $13(61.9)$ & \\
\hline Tumor grade & & & 0.082 & & & 0.88 \\
\hline 1 & $157(31.8)$ & $336(68.2)$ & & $197(40.0)$ & $296(60.0)$ & \\
\hline $2+3$ & $122(37.8)$ & $201(62.2)$ & & $127(39.3)$ & $196(60.7)$ & \\
\hline Unknown & $28(38.4)$ & $45(61.6)$ & & $31(42.5)$ & $42(57.5)$ & \\
\hline Estrogen receptor & & & 0.38 & & & 0.49 \\
\hline Positive & $236(33.8)$ & $463(66.2)$ & & $285(40.8)$ & $414(59.2)$ & \\
\hline Negative & $66(37.3)$ & $111(62.7)$ & & $67(37.9)$ & $110(62.2)$ & \\
\hline Unknown & $5(38.5)$ & $8(61.5)$ & & $3(23.1)$ & $10(76.9)$ & \\
\hline HER2 status & & & 0.93 & & & 0.79 \\
\hline Negative & $255(34.6)$ & $482(65.4)$ & & $296(40.2)$ & $441(59.8)$ & \\
\hline Positive & $52(34.2)$ & $100(65.8)$ & & $59(38.8)$ & $93(61.2)$ & \\
\hline Ki-67 expression level ${ }^{\mathrm{c}}$ & & & 0.044 & & & 0.037 \\
\hline Low & $131(30.4)$ & $300(69.6)$ & & $192(44.6)$ & $239(55.5)$ & \\
\hline High & $137(37.1)$ & $232(62.9)$ & & $137(37.1)$ & $232(62.9)$ & \\
\hline Unknown & $39(43.8)$ & $50(56.2)$ & & $26(29.2)$ & $63(70.8)$ & \\
\hline Chemotherapy & & & 0.080 & & & 0.18 \\
\hline Yes & $133(38.0)$ & $217(62.0)$ & & $130(37.1)$ & $220(62.9)$ & \\
\hline No & $174(32.3)$ & $365(67.7)$ & & $225(41.7)$ & $314(58.3)$ & \\
\hline
\end{tabular}

${ }^{\mathrm{a} H i g h:} \geq 2.72$, low: $<2.72$; ${ }^{\mathrm{b}} \mathrm{High}:>1,688 / \mu 1$, low: $\leq 1,688 / \mu 1$; ${ }^{\mathrm{c}}$ Low: $<20 \%$, high: $\geq 20 \%$. ALC, absolute lymphocyte count; HER2, human epidermal growth factor receptor type 2; NLR, neutrophil-to-lymphocyte ratio.

the operation. For patients treated with preoperative chemotherapy, this data was obtained using blood samples collected just before the start of the treatment. Neutrophil and lymphocyte counts in peripheral blood were measured automatically using Sysmex XN-9000 or XN-1000 hematology analyzers (Sysmex Corporation, Kobe, Japan). Briefly, each blood sample was treated with reagents provided with the kit that can distinguish subsets of white blood cells, including neutrophils and lymphocytes, and each fraction was separated by flow cytometry (18). The NLR was calculated by dividing the number of neutrophils by the number of lymphocytes. ALC was obtained by multiplying total white blood cell counts with the percentage of lymphocytes.

Statistical analysis. The cut-off value of NLR for RFS was calculated from the receiver operating characteristic (ROC) curve using the Youden index for areas under the curve (AUC).
Relationships between NLR levels and clinical factors were compared using the Chi-square or Fisher's exact tests, as appropriate. Kaplan-Meier plots of RFS or OS in the separate groups were calculated using log-rank tests. Univariate and multivariate analyses of clinicopathological factors and NLR were done using a Cox proportional-hazards model to obtain the HR and $95 \%$ CI. Statistical significance was set at $\mathrm{P}<0.05$ and the statistical calculations were performed using $\mathrm{JMP}^{\circledR}$, v.12 (SAS Institute, Inc., Cary, NC, USA).

\section{Results}

Determination of the baseline NLR cut-off value for RFS. The cut-off value of NLR for RFS was determined based on the ROC curve calculated using the Youden index for AUC and set at 2.72 (AUC: 0.566, sensitivity: 0.466, specificity: 0.668, 
Table II. Univariate and multivariate analyses of RFS.

\begin{tabular}{|c|c|c|c|c|c|}
\hline Variable & $\mathrm{n}$ & $\begin{array}{c}\text { Univariate analysis, } \\
\text { HR }(95 \% \mathrm{CI})^{\mathrm{a}}\end{array}$ & P-value & $\begin{array}{c}\text { Multivariate analysis, } \\
\text { HR }(95 \% \mathrm{CI})^{\mathrm{a}}\end{array}$ & P-value \\
\hline Menopausal status & & & 0.13 & & \\
\hline Pre- & 308 & 1.00 & & & \\
\hline Post- & 573 & $1.43(0.90-2.36)$ & & & \\
\hline Tumor size, cm & & & $<0.0001$ & & 0.0009 \\
\hline$\leq 2.0$ & 526 & 1.00 & & 1.00 & \\
\hline$>2$ & 358 & $2.58(1.67-4.04)$ & & $2.35(1.45-4.53)$ & \\
\hline Lymph node metastasis & & & $<0.0001$ & & 0.0065 \\
\hline Negative & 596 & 1.00 & & 1.00 & \\
\hline Positive & 272 & $3.06(1.97-4.78)$ & & $2.08(1.23-3.54)$ & \\
\hline Tumor grade & & & 0.0012 & & 0.37 \\
\hline 1 & 493 & 1.00 & & 1.00 & \\
\hline $2+3$ & 323 & $2.07(1.33-3.23)$ & & $1.30(0.73-2.32)$ & \\
\hline Estrogen receptor status & & & 0.039 & & 0.58 \\
\hline Positive & 699 & 1.00 & & 1.00 & \\
\hline Negative & 177 & $1.67(1.03-2.64)$ & & $1.21(0.61-2.26)$ & \\
\hline HER2 status & & & 0.96 & & \\
\hline Negative & 737 & 1.00 & & & \\
\hline Positive & 152 & $0.96(0.53-1.63)$ & & & \\
\hline Ki-67 expression level ${ }^{\mathrm{b}}$ & & & $<0.0001$ & & 0.012 \\
\hline Low & 431 & 1.00 & & 1.00 & \\
\hline High & 369 & $2.64(1.64-4.36)$ & & $2.25(1.20-4.26)$ & \\
\hline Chemotherapy & & & 0.043 & & 0.17 \\
\hline No & 539 & 1.00 & & 1.00 & \\
\hline Yes & 350 & $1.56(1.02-2.41)$ & & $0.68(0.39-1.18)$ & \\
\hline NLR level $^{\mathrm{c}}$ & & & 0.047 & & 0.14 \\
\hline Low & 582 & 1.00 & & 1.00 & \\
\hline High & 307 & $1.56(1.01-2.39)$ & & $1.46(0.89-2.39)$ & \\
\hline ALC level $^{\mathrm{d}}$ & & & 0.37 & & \\
\hline Low & 534 & 1.00 & & & \\
\hline High & 355 & $1.22(0.79-1.88)$ & & & \\
\hline
\end{tabular}

${ }^{\mathrm{a}} \mathrm{HR}$ (95\% CI); 'bow: $<20 \%$, high: $\geq 20 \%$; ${ }^{\mathrm{c} H i g h: ~} \geq 2.72$, low: $<2.72$; ${ }^{\mathrm{d}} \mathrm{High}:>1,688 / \mu 1$, low: $\leq 1,688 / \mu 1$. HR, hazard ratio; ALC, absolute lymphocyte count; CI, confidence interval; NLR, neutrophil-to-lymphocyte ratio; RFS, recurrence-free survival.

$\mathrm{P}=0.1598)$ as shown in the Fig. S1. According to this cut-off value, 889 patients were classified into two groups: NLR-high $(\mathrm{n}=307)$ and -low $(\mathrm{n}=582)$.

Relationship of NLR levels with RFS or OS according to ALC levels. The RFS of NLR-low patients was significantly better than that in NLR-high patients $(\mathrm{P}=0.036$, Fig. 1A). On the contrary, there was no significant correlation between OS and NLR levels ( $\mathrm{P}=0.24$, Fig. 1B). Next, we classified patients into five groups with an equal number of patients to their ALC; the HRs and 95\% CIs of NLR levels for RFS in each ALC group are shown in Fig. 2. The RFS of patients with NLR-low and -high was similar in the three groups with the lowest ALCs, namely ALC-1 ( $\leq 1149 / \mu 1$; HR, 0.85; 95\% CI: 0.28-2.21; $\mathrm{n}=178)$, ALC-2 (>1149/ $\mu 1, \leq 1398 / \mu 1 ;$ HR, $1.04 ; 95 \%$ CI: 0.39-2.93; $\mathrm{n}=178)$, and ALC-3 (>1398/ $\mu 1, \leq 1688 / \mu 1 ; \mathrm{HR}, 0.68 ; 95 \% \mathrm{CI}$ :
0.22-2.11; $n=178)$. On the contrary, the RFS of patients with NLR-low was better than that with NLR-high in the second highest (ALC-4; $\mathrm{n}=178 ;>1688 / \mu 1, \leq 1977 / \mu 1 ; \mathrm{HR}, 0.18 ; 95 \% \mathrm{CI}$ : 0.07-0.45) and highest (ALC-5; $\mathrm{n}=177 ;>1977 / \mu 1 ; \mathrm{HR}, 0.33$; 95\% CI: 0.09-2.14) groups.

Based on these results, we further divided all the patients into either ALC-low ( $\leq 1688 / \mu 1 ; \mathrm{n}=534)$ and -high (>1688/ $\mu 1$; $\mathrm{n}=355$ ) groups. There was no significant association between ALC and RFS ( $\mathrm{P}=0.37$; Fig. $3 \mathrm{~A})$ or $\mathrm{OS}(\mathrm{P}=0.78$; Fig. $3 \mathrm{~B})$. We further analyzed the prognostic significance of NLR according to the ALC. There was no significant association between NLR and RFS in the ALC-low group ( $\mathrm{P}=0.46$, Fig. 4A). In contrast, the RFS of NLR-low patients (5-year RFS, $0.88 ; n=317)$ was significantly better than that of NLR-high patients (5-year RFS, 0.57; $\mathrm{n}=38$; $\mathrm{P}<0.0001$, Fig. 4B) in the ALC-high group. Similarly, OS was 
Table III. Univariate and multivariate analyses of OS.

\begin{tabular}{|c|c|c|c|c|c|}
\hline Variable & $\mathrm{n}$ & $\begin{array}{c}\text { Univariate analysis, } \\
\text { HR }(95 \% \mathrm{CI})^{\mathrm{a}}\end{array}$ & P-value & $\begin{array}{l}\text { Multivariate analysis, } \\
\qquad \mathrm{HR}(95 \% \mathrm{CI})^{\mathrm{a}}\end{array}$ & P-value \\
\hline Menopausal status & & & 0.0061 & & 0.0037 \\
\hline Pre- & 308 & 1.00 & & 1.00 & \\
\hline Post- & 573 & $2.97(1.33-7.89)$ & & $3.51(1.46-10.39)$ & \\
\hline Tumor size, $\mathrm{cm}$ & & & 0.0046 & & 0.16 \\
\hline$\leq 2.0$ & 526 & 1.00 & & 1.00 & \\
\hline$>2$ & 358 & $1.90(1.01-3.64)$ & & $1.69(0.81-3.67)$ & \\
\hline Lymph node metastasis & & & 0.0003 & & 0.0023 \\
\hline Negative & 596 & 1.00 & & 1.00 & \\
\hline Positive & 272 & $3.42(1.76-6.87)$ & & $3.12(1.50-6.78)$ & \\
\hline Tumor grade & & & 0.13 & & \\
\hline 1 & 493 & 1.00 & & & \\
\hline $2+3$ & 323 & $1.66(0.86-3.19)$ & & & \\
\hline Estrogen receptor status & & & 0.033 & & 0.20 \\
\hline Positive & 699 & 1.00 & & 1.00 & \\
\hline Negative & 177 & $2.14(1.07-4.10)$ & & $1.72(0.73-3.38)$ & \\
\hline HER2 status & & & 0.59 & & \\
\hline Negative & 737 & 1.00 & & & \\
\hline Positive & 152 & $0.79(0.30-1.76)$ & & & \\
\hline Ki-67 expression level ${ }^{\mathrm{b}}$ & & & $<0.0001$ & & 0.0037 \\
\hline Low & 431 & 1.00 & & 1.00 & \\
\hline High & 369 & $4.02(1.98-8.83)$ & & $3.51(1.46-10.39)$ & \\
\hline Chemotherapy & & & 0.13 & & \\
\hline No & 539 & 1.00 & & & \\
\hline Yes & 350 & $1.62(0.86-2.41)$ & & & \\
\hline NLR level $^{\mathrm{c}}$ & & & 0.23 & & \\
\hline Low & 582 & 1.00 & & & \\
\hline High & 307 & $1.47(0.78-2.77)$ & & & \\
\hline $\mathrm{ALC} \mathrm{level}^{\mathrm{d}}$ & & & 0.78 & & \\
\hline Low & 534 & 1.00 & & & \\
\hline High & 355 & $0.91(0.46-1.73)$ & & & \\
\hline
\end{tabular}

${ }^{\mathrm{a}} \mathrm{HR}$ (95\% CI); ${ }^{\mathrm{b}} \mathrm{How}:<20 \%$, high: $\geq 20 \%$; ${ }^{\mathrm{c} H i g h: ~} \geq 2.72$, low: $<2.72$; ${ }^{\mathrm{d}} \mathrm{High}:>1,688 / \mu 1$, low: $\leq 1,688 / \mu 1$. HR, hazard ratio; ALC, absolute lymphocyte count; CI, confidence interval; NLR, neutrophil-to-lymphocyte ratio; OS, overall survival.

significantly better in the NLR-low subset than that in the NLR-high subset $(\mathrm{P}=0.015$; Fig. 4D) in the ALC-high group, but we found no significant association between NLR and OS in the ALC-low group ( $\mathrm{P}=0.99 ;$ Fig. 4C).

Correlation between the clinicopathological characteristics and NLR or ALC. Significantly more NLR-high patients were found to be premenopausal $(\mathrm{P}=0.022)$, and to have large tumor size $(\mathrm{P}=0.021)$ and high expression of $\mathrm{Ki}-67(\mathrm{P}=0.044)$, as shown in Table I. There was no significant association between the NLR and other clinicopathological factors, including lymph node metastasis, tumor grade, ER, HER2, and chemotherapy administration. The frequency of patients with ALC-high was significantly higher in the postmenopausal group $(\mathrm{P}=0.0012)$, and in the Ki-67-low group ( $\mathrm{P}=0.037)$.
Univariate and multivariate analyses of RFS and OS. According to univariate analysis, tumor size $(\mathrm{P}<0.0001)$, lymph node metastasis $(\mathrm{P}<0.0001)$, tumor grade $(\mathrm{P}=0.0012)$, ER status $(\mathrm{P}=0.039), \mathrm{Ki}-67$ expression level $(\mathrm{P}<0.0001)$, chemotherapy administration $(\mathrm{P}=0.043)$ and NLR $(\mathrm{P}=0.047)$, but not ALC $(\mathrm{P}=0.37)$ were significant prognostic factors for RFS, as shown in Table II. Among these parameters, tumor size $(\mathrm{P}=0.0009)$, lymph node metastasis $(\mathrm{P}=0.0065)$ and $\mathrm{Ki}-67$ expression level $(\mathrm{P}=0.012)$, but not NLR $(\mathrm{P}=0.14)$, were significant and independent prognostic factors for RFS. Similarly, menopausal status $(\mathrm{P}=0.0037)$, lymph node metastasis $(\mathrm{P}=0.0023)$ and Ki-67 ( $\mathrm{P}=0.0037)$ were identified as significant and independent factors for OS by multivariate analysis (Table III). However, no significant association between NLR or ALC $(\mathrm{P}=0.78)$ and OS was found by univariate analysis. 
Table IV. Univariate and multivariate analyses of RFS in the ALC-high subgroup ${ }^{\mathrm{a}}$.

\begin{tabular}{|c|c|c|c|c|c|}
\hline Variable & $\mathrm{n}$ & $\begin{array}{l}\text { Univariate analysis, } \\
\text { HR }(95 \% \mathrm{CI})^{\mathrm{b}}\end{array}$ & P-value & $\begin{array}{l}\text { Multivariate analysis, } \\
\text { HR }(95 \% \mathrm{CI})^{\mathrm{b}}\end{array}$ & P-value \\
\hline Menopausal status & & & 1.00 & & \\
\hline Pre- & 100 & 1.00 & & & \\
\hline Post- & 251 & $1.00(0.49-2.19)$ & & & \\
\hline Tumor size, cm & & & 0.0003 & & \\
\hline$\leq 2.0$ & 207 & 1.00 & & 1.00 & 0.065 \\
\hline$>2$ & 147 & $3.49(1.75-7.43)$ & & $2.17(0.95-5.30)$ & \\
\hline Lymph node metastasis & & & 0.0003 & & 0.018 \\
\hline Negative & 244 & 1.00 & & 1.00 & \\
\hline Positive & 103 & $3.60(1.81-7.32)$ & & $2.54(1.18-5.68)$ & \\
\hline Tumor grade & & & 0.40 & & \\
\hline 1 & 197 & 1.00 & & & \\
\hline $2+3$ & 127 & $1.35(0.66-2.70)$ & & & \\
\hline Ki-67 expression level ${ }^{\mathrm{c}}$ & & & 0.0039 & & 0.073 \\
\hline Low & 192 & 1.00 & & 1.00 & \\
\hline High & 137 & $2.78(1.38-5.83)$ & & $2.07(0.94-4.77)$ & \\
\hline Chemotherapy & & & 0.018 & & 0.98 \\
\hline No & 225 & 1.00 & & 1.00 & \\
\hline Yes & 130 & $2.24(1.15-4.45)$ & & $1.01(0.45-2.35)$ & \\
\hline NLR level $^{\mathrm{d}}$ & & & 0.0002 & & 0.0023 \\
\hline Low & 317 & 1.00 & & 1.00 & \\
\hline High & 38 & $4.28(2.06-8.45)$ & & $3.52(1.61-7.32)$ & \\
\hline
\end{tabular}

${ }^{\mathrm{a} H i g h}$ : $>1,688 / \mu 1$, low: $\leq 1,688 / \mu 1$; ${ }^{\text {bHR }}(95 \% \mathrm{CI})$; ${ }^{\mathrm{L}} \mathrm{Low}$ : $<20 \%$, high: $\geq 20 \%$; ${ }^{\mathrm{d}} \mathrm{High}: \geq 2.72$, low: $<2.72$. HR, hazard ratio; CI, confidence interval; NLR, neutrophil-to-lymphocyte ratio; RFS, recurrence-free survival; ALC, absolute lymphocyte count.

Next, we calculated the prognostic significance of clinical parameters in ALC-high breast cancer subgroup. Multivariate analysis revealed that lymph node metastasis (HR 2.54; 95\% CI: 1.18-5.68; $\mathrm{P}=0.018$ ), and NLR (HR: 3.52, 95\% CI: $1.61-7.32, \mathrm{P}=0.0023$ ) associated significantly and independently with RFS (Table IV). Significant and marginally significant associations were observed in lymph node metastasis (HR 4.60; 95\% CI 1.21-22.4; $\mathrm{P}=0.024$ ) and NLR (HR: 3.41, 95\% CI: 0.90-11.7, $\mathrm{P}=0.070$ ), respectively, for OS by multivariate analysis (Table $\mathrm{V}$ ).

\section{Discussion}

In the present study, we demonstrated that the RFS of NLR-low patients was significantly better than that of NLR-high patients. This prognostic impact of NLR is exclusive among patients with a high-ALC. Although multivariate analysis showed that NLR was not a significant prognostic factor for RFS in all breast cancers, NLR was found to be a significant and independent prognostic factor for RFS in patients with high ALC. It is well established that pretreatment NLR is a significant predictor of prognosis in operated breast cancer patients, with reported HR of DFS in NLR-high patients ranging from 1.62 to $1.80(2,3)$. We consistently obtained similar HR of RFS $(1.56$; $95 \% \mathrm{CI}$ : 1.01-2.39) in total patients, but found that the HR increased to 3.52 (95\% CI: 1.61-7.32) in the subgroup with high ALC. To the best of our knowledge, ours is the first report to analyze NLR in association with the absolute counts of peripheral lymphocytes.

Since local immunoreaction against cancer cells seems to be modulated by TILs, elevated levels of TILs in tumors lead to favorable prognoses. On the other hand, the presence of neutrophils in tumors contributes to cancer proliferation and invasion ability, mediated by their induction of cytokines and chemokines $(5,6)$. The association between high NLR and poor prognoses could be explained on the basis of these speculated lymphocyte and neutrophil functions. Interestingly, neither absolute neutrophil (data not shown) nor ALCs (Fig. 3), but rather the ratio between these parameters (Fig. 1), was significantly associated with patient prognoses. These data suggest that direct or indirect interactive effects of lymphocytes and neutrophils on tumor progression influence patient prognoses, although the mechanistic details of such interactions remain unclear.

Details of the usefulness of NLR as a prognostic indicator for breast cancer patients are currently unknown. It is reasonable to consider that local immunoreactions against cancer cells, evaluable by TILs result in a higher proportion of lymphocytes in the blood. However, NLR and TILs may not necessarily be synonymous. Intra-tumoral infiltrating lymphocyte subgroups and NLR are independent prognostic factors in non-small cell lung cancer (19). In addition, no 
Table V. Univariate and multivariate analyses of OS in the ALC-high subgroup ${ }^{\mathrm{a}}$.

\begin{tabular}{|c|c|c|c|c|c|}
\hline Variable & $\mathrm{n}$ & $\begin{array}{l}\text { Univariate analysis, } \\
\text { HR }(95 \% \mathrm{CI})^{\mathrm{b}}\end{array}$ & P-value & $\begin{array}{c}\text { Multivariate analysis, } \\
\text { HR }(95 \% \mathrm{CI})^{\mathrm{b}}\end{array}$ & P-value \\
\hline Menopausal status & & & 0.044 & & \\
\hline Pre- & 100 & 1.00 & & & \\
\hline Post- & 251 & $5.28(1.04-96.3)$ & & & \\
\hline Tumor size, $\mathrm{cm}$ & & & 0.024 & & 0.33 \\
\hline$\leq 2.0$ & 207 & 1.00 & & 1.00 & \\
\hline$>2$ & 147 & $3.54(1.18-13.0)$ & & $1.98(0.51-9.76)$ & \\
\hline Lymph node metastasis & & & 0.0030 & & 0.024 \\
\hline Negative & 244 & 1.00 & & 1.00 & \\
\hline Positive & 103 & $6.46(1.86-29.6)$ & & $4.60(1.21-22.4)$ & \\
\hline Tumor grade & & & 0.60 & & \\
\hline 1 & 197 & 1.00 & & & \\
\hline $2+3$ & 127 & $0.73(0.19-2.32)$ & & & \\
\hline Ki-67 expression level ${ }^{\mathrm{d}}$ & & & 0.024 & & 0.11 \\
\hline Low & 192 & 1.00 & & 1.00 & \\
\hline High & 137 & $3.50(1.18-11.6)$ & & $2.68(0.81-10.3)$ & \\
\hline Chemotherapy & & & 0.17 & & \\
\hline No & 225 & 1.00 & & & \\
\hline Yes & 130 & $2.10(0.73-6.41)$ & & & \\
\hline NLR level $^{\mathrm{e}}$ & & & 0.035 & & 0.070 \\
\hline Low & 317 & 1.00 & & 1.00 & \\
\hline High & 38 & $3.69(1.11-10.9)$ & & $3.41(0.90-11.7)$ & \\
\hline
\end{tabular}

${ }^{\mathrm{a}} \mathrm{High}:>1,688 / \mu 1 ;{ }^{\mathrm{b}} \mathrm{HR}(95 \% \mathrm{CI})$; ${ }^{\mathrm{C}}$ Not included in multivariate analysis due to error; ${ }^{\mathrm{d}}$ Low: $<20 \%$, high: $\geq 20 \%$; ${ }^{\mathrm{e}} \mathrm{High}: \geq 2.72$, low: $<2.72$. HR, hazard ratio; CI, confidence interval; NLR, neutrophil-to-lymphocyte ratio; OS, overall survival; ALC, absolute lymphocyte count.

correlation was determined between TILs and NLR in gastric cancer (20), and there was no significant association between percentage changes of TILs and NLR in triple negative breast cancers after treatment with neoadjuvant chemotherapy (21). Since the prognostic importance of baseline NLR in patients receiving immune checkpoint inhibitors was demonstrated in a meta-analysis (22), data from the studies mentioned above seem to indicate that NLR reflects cancer immunity differently from that evaluable by TILs in each patient.

In the present study, we examined the prognostic significance of the NLR according to ALC levels in Fig. 2 and demonstrated that among patients with ALC-low, NLR did not influence patient prognosis. However, the RFS of patients with low NLR was significantly better than that with high NLR in the ALC-high subgroup which exceeded $1,688 / \mu 1$. Our observations may indicate that even among patients with ALC-high, unfavorable effects seem to be induced by neutrophils. On the contrary, no indications for prognosis could be obtained by neutrophils in patients with low levels of ALC. Neutrophils play important roles in the development of breast cancer cells, mediated through metalloproteinase-9 (MMP-9) and other factors which that promote cell proliferation, inhibition of apoptosis, and angiogenesis $(23,24)$. In addition, suppressive functions of T cell immunity against cancer cells by neutrophils are reported (25). These functions, which promote cancer proliferation and progression, seem to abolish lymphocyte-induced adaptive immune reactions. As mentioned previously, no effect of the immune checkpoint inhibitor was observed in patients with a low baseline ALC $(11,12)$. This may indicate that there is very little contribution to cancer immunity induced by neutrophils in patients with low ALC. We speculate that the negative effect of neutrophils is related to the process of cancer immunity mediated through lymphocytes and thus, the prognostic impact was observed exclusively in patients with ALC-high.

In the current study, high NLR was significantly more frequent among premenopausal patients, and in those with large tumor sizes and high levels of Ki-67. However, high ALC was significantly frequent in postmenopausal patients and in patients with breast cancers with low Ki-67 (Table I). Similar to our observations, higher NLR has been found to be associated with younger age and increased T stage (26). In spite of the link between these clinical factors, NLR was confirmed by multivariate analysis including tumor size, lymph node metastasis, chemotherapy use, and Ki-67 expression levels to be a significant and independent prognostic factor in breast cancer with ALC-high (Table IV). Positive correlations between good response to neoadjuvant chemotherapy and low NLR have been reported $(27,28)$. However, significant increase in RFS in patients with low NLR in the ALC-high group was recognized irrespective of neoadjuvant or adjuvant chemotherapy. We think this parameter is prognostic rather than predictive for chemotherapy in operated breast cancers. 
The limitations of this study were that the cut-off value of NLR at 2.72 was determined on the basis of ROC analysis. In a previous study, several NLR cut-off values were used, varying from 1.81 to 4 (3). Furthermore, the cut-off value of ALC, which distinguishes the usefulness of NLR in predicting prognosis, was obtained by dividing the patients into five groups. The HRs of NLR for RFS were consistently close to one in the first three subgroups ALC-1, -2, and -3. Since the HRs were less than 0.5 in the remaining two subgroups (ALC-4 and -5), the cut-off of ALC was set at 1,688/ $\mu 1$. The optimal NLR and ALC cut-off values for prognosis remains to be identified. Another limitation was that we had insufficient data concerning the relationship between TILs and NLR, and the follow-up time (median, 30.7 months) is relatively short. The results obtained in the present study concerning TILs require further evaluation in future studies including larger numbers of patients with a longer follow-up.

In conclusion, we have demonstrated that low NLR is a significant prognostic factor for RFS of breast cancer patients with ALC-high. The novelty of the present study is that we were able to demonstrate the prognostic usefulness of NLR exclusively in the ALC - high subgroup. This is the first study to demonstrate that the prognostic usefulness of NLR is restricted in the subgroup of ALC-high. Since NLR and ALC are measured in daily clinical practice, these findings may contribute to a better understanding of the mechanisms underlying NLR, and will be useful for identifying patients with high recurrence risk more accurately who need additional treatments.

\section{Acknowledgements}

Not applicable.

\section{Funding}

The present study was supported by a Grant-in-Aid from the Ministry of Education, Culture, Sports, Science, and Technology of Japan (grant no. 15K10077).

\section{Availability of data and materials}

The datasets analyzed during the current study are not publicly available as the Ethics Committee of Hyogo College of Medicine does not have permission to provide the data of individual participants, but they are available from the corresponding author on reasonable request.

\section{Authors' contributions}

YF analyzed and interpreted the data, and drafted the manuscript. YaM contributed to the conception and design of the study, and revised the manuscript. HO, TH, YoM, AB and MI acquired and interpreted the data. All of the authors approved the final manuscript and agreed to be accountable for all aspects of the work.

\section{Ethics and approval and consent to participate}

The present study was approved by the Ethics Committee of the Hyogo College of Medicine (Hyogo, Japan; no. 1886) in accordance with the Declaration of Helsinki. As the present study collected only retrospective clinical data and offered no risk to the participants, written informed consent was not required.

\section{Patient consent for publication}

Not applicable.

\section{Competing interests}

All authors declare that they have no competing interests.

\section{References}

1. Jia W, Wu J, Jia H, Yang Y, Zhang X, Chen K and Su F: The peripheral blood neutrophil-to-lymphocyte ratio is superior to the lymphocyte-to-monocyte ratio for predicting the long-term survival of triple-negative breast cancer patients. PLoS One 10: e0143061, 2015.

2. Ethier JL, Desautels D, Templeton A, Shah PS and Amir E: Prognostic role of neutrophil-to-lymphocyte ratio in breast cancer: A systematic review and meta-analysis. Breast Cancer Res 19: 2, 2017.

3. Liu X, Qu JK, Zhang J, Yan Y, Zhao XX, Wang JZ, Qu HY, Liu L, Wang JS and Duan XY: Prognostic role of pretreatment neutrophil to lymphocyte ratio in breast cancer patients: A meta-analysis. Medicine (Baltimore) 96: e8101, 2017.

4. Savas P, Salgado R, Denkert C, Sotiriou C, Darcy PK, Smyth MJ and Loi S: Clinical relevance of host immunity in breast cancer: From TILs to the clinic. Nat Rev Clin Oncol 13: 228-241, 2016.

5. Landskron G, De la Fuente M, Thuwajit P, Thuwajit C and Hermoso MA: Chronic inflammation and cytokines in the tumor microenvironment. J Immunol Res 2014: 149185, 2014.

6. Powell DR and Huttenlocher A: Neutrophils in the tumor microenvironment. Trends Immunol 37: 41-52, 2016.

7. Goldhirsch A, Ingle JN, Gelber RD, Coates AS, Thrülimann B, Senn HJ; Panel members: Thresholds for therapies: Highlights of the St Gallen International Expert Consensus on the primary therapy of early breast cancer 2009. Ann Oncol 20: 1319-1329, 2009.

8. Wang K, Xu J, Zhang T, Xue D. Tumor-infiltrating lymphocytes in breast cancer predict the response to chemotherapy and survival outcome: A meta-analysis. Oncotarget 7: 44288-44298, 2016.

9. Stanton SE, Disis ML. Clinical significance of tumor-infiltrating lymphocytes in breast cancer. J Immunother Cancer 4: 59, 2016.

10. Iwase T, Sangai T, Sakakibara M, Sakakibara J, Ishigami E, Hayama S, Nakagawa A, Masuda T, Tabe S and Nagashima T: An increased neutrophil-to-lymphocyte ratio predicts poorer survival following recurrence for patients with breast cancer. Mol Clin Oncol 6: 266-270, 2017.

11. Diehl A, Yarchoan M, Hopkins A, Jaffee E and Grossman SA: Relationships between lymphocyte counts and treatment-related toxicities and clinical responses in patients with solid tumors treated with PD-1 checkpoint inhibitors. Oncotarget 8: 114268-114280, 2017.

12. Tanizaki J, Haratani K, Hayashi H, Chiba Y, Nakamura Y, Yonesaka K, Kudo K, Kaneda H, Hasegawa Y, Tanaka K, et al: Peripheral blood biomarkers associated with clinical outcome in non-small cell lung cancer patients treated with nivolumab. J Thorac Oncol 13: 97-105, 2018.

13. Goldhirsch A, Glick JH, Gelber RD, Coates AS, Thürlimann B and Senn HJ; Panel members: Meeting highlights: International expert consensus on the primary therapy of early breast cancer 2005. Ann Oncol 16: 1569-1583, 2005.

14. Goldhirsch A, Wood WC, Gelber RD, Coates AS, Thürlimann B and Senn HJ: Progress and promise: Highlights of the international expert consensus on the primary therapy of early breast cancer 2007. Ann Oncol 18: 1133-1144, 2007.

15. Goldhirsch A, Wood WC, Coates AS, Gelber RD, Thrülimann B and Senn HJ; Panel members: Strategies for subtypes-dealing with the diversity of breast cancer: Highlights of the St. Gallen International Expert Consensus on the primary therapy of early breast cancer. Ann Oncol 22: 1736-1747, 2011. 
16. Goldhirsch A,WinerEP,Coates AS, Gelber RD,Piccart-GebhartM, Thürlimann B, Senn HJ; Panel members: Personalizing the treatment of women with early breast cancer: Highlights of the St Gallen International Expert Consensus on the primary therapy of early breast cancer 2013. Ann Oncol 24: 2206-2223, 2013.

17. Coates AS, Winer EP, Goldhirsch A, Gelber RD, Gnant M, Piccart-Gebhart M, Thürlimann B and Senn HJ; Panel Members: Tailoring therapies-improving the management of early breast cancer: St Gallen International Expert Consensus on the primary therapy of early breast cancer 2015. Ann Oncol 26: 1533-1546, 2015

18. Kawauchi S, Kono M, Takagi Y, Wada A and Morikawa T: The positions of normal leukocytes on the scattergram of the newly developed abnormal cell-detection channel of the $\mathrm{XN}$-series multi-parameter automated hematology analyzers. Sysmex J Int 23: 1-9, 2013

19. Dirican N, Karakaya YA, Gunes S, Daloglu FT and Dirican A Association of intra-tumoral tumour-infiltrating lymphocytes and neutrophil-to-lymphocyte ratio is an independent prognostic factor in non-small cell lung cancer. Clin Respir J 11: 789-796, 2017.

20. Dirican A, Ekinci N, Avci A, Akyol M, Alacacioglu A Kucukzeybek Y, Somali I, Erten C, Demir L, Can A, et al: The effects of hematological parameters and tumor-infiltrating lymphocytes on prognosis in patients with gastric cancer. Cancer Biomark 13: 11-20, 2013.

21. Lee J, Kim DM and Lee A: Prognostic role and clinical association of tumor-infiltrating lymphocyte, programmed death ligand-1 expression with neutrophil-lymphocyte ratio in locally advanced triple-negative breast cancer. Cancer Res Treat, doi:https://doi.org/10.4143/crt.2018.270.
22. Sacdalan DB, Lucero JA and Sacdalan DL: Prognostic utility of baseline neutrophil-to-lymphocyte ratio in patients receiving immune checkpoint inhibitors: A review and meta-analysis. Onco Targets Ther 11: 955-965, 2018.

23. Houghton AM: The paradox of tumor-associated neutrophils: Fueling tumor growth with cytotoxic substances. Cell Cycle 9: 1732-1737, 2010.

24. Hurt B, Schulick R, Edil B, El Kasmi KC and Barnett C Jr: Cancer-promoting mechanisms of tumor-associated neutrophils. Am J Surg 214: 938-944, 2017.

25. Uribe-Querol E and Rosales C: Neutrophils in cancer: Two sides of the same coin. J Immunol Res 2015: 983698, 2015.

26. Noh H, Eomm M and Han A: Usefulness of pretreatment neutrophil to lymphocyte ratio in predicting disease-specific survival in breast cancer patients. J Breast Cancer 16: 55-59, 2013.

27. Chen Y, Chen K, Xiao X, Nie Y, Qu S, Gong C, Su F and Song E: Pretreatment neutrophil-to-lymphocyte ratio is correlated with response to neoadjuvant chemotherapy as an independent prognostic indicator in breast cancer patients: A retrospective study. BMC Cancer 16: 320, 2016.

28. Asano Y, Kashiwagi S, Onoda N, Noda S, Kawajiri H, Takashima T, Ohsawa M, Kitagawa $\mathrm{S}$ and Hirakawa K: Predictive value of neutrophil/lymphocyte ratio for efficacy of preoperative chemotherapy in triple-negative breast cancer. Ann Surg Oncol 23: 1104-1110, 2016. 